\title{
Expression of miR-26b in ovarian carcinoma tissues and its correlation with clinicopathology
}

\author{
JIANJUN LU, WEI ZHANG, YANG DING, XIANG LI and JIANDONG SONG \\ Department of Gynaecology and Obstetrics, Affiliated Hospital of Inner Mongolia Medical University, \\ Hohhot, Inner Mongolia 010059, P.R. China
}

Received September 24, 2018; Accepted February 12, 2019

DOI: $10.3892 / \mathrm{ol} .2019 .10117$

\begin{abstract}
The expression of microRNA (miR)-26b in ovarian carcinoma tissues, its correlation with clinicopathology, and its effect on diagnostic value and prognosis of ovarian cancer was investigated. A total of 74 patients with ovarian cancer (the study group) and 30 patients with benign ovarian tumors (the control group) in the Affiliated Hospital of Inner Mongolia Medical University from July 2011 to June 2013 were retrospectively analyzed. The expression of miR-26b in ovarian carcinoma tissues was detected by fluorescence reverse transcription-quantitative polymerase chain reaction (RT-qPCR), and the correlation between the expression of miR-26b and the pathological features of ovarian carcinoma tissues and prognosis of patients was analyzed. The expression level of miR-26b in the study group $(0.28 \pm 0.07)$ was significantly lower than that in the control group $(0.54 \pm 0.11$; $\mathrm{P}<0.050)$. There was no significant correlation between miR-26b expression and age, tumor type, exercise habit, smoking habit of patients with ovarian cancer $(\mathrm{P}>0.050)$, but there was close correlation between the miR-26b expression and lymph node metastasis, differentiation degree and pathological stage of patients with ovarian cancer $(\mathrm{P}<0.001)$. ROC curve showed that the area under curve (AUC) was 0.839, and when the maximum cut-off value was 0.815 , the sensitivity and specificity of miR-26b in diagnosing the ovary was $84.932 \%$ and $77.936 \%$, respectively. The 5-year overall survival rate in the low-expression group (61.54\%) was significantly lower than that in the high-expression group $(84.85 ; \mathrm{P}=0.028)$. miR-26b is under-expressed in the ovary and has a close relationship with pathological stage, differentiation degree, and lymph node metastasis of ovarian cancer, which indicates that miR-26b is involved in the occurrence and development of ovarian cancer and is expected to be an effective indicator for treatment and diagnosis of ovarian cancer and the prognosis of patients.
\end{abstract}

Correspondence to: Dr Jiandong Song, Department of Gynaecology and Obstetrics, Affiliated Hospital of Inner Mongolia Medical University, 1 Tongdao North street, Huimin, Hohhot, Inner Mongolia 010059, P.R. China

E-mail: songjdnm@163.com

Key words: miR-26b, ovarian cancer, clinicopathology, prognosis

\section{Introduction}

Ovarian cancer is one of the most common malignant tumors in female genital organs, and also one of the types of tumor with high incidence rate among females (1). Statistics show that there are more than 300,000 new ovarian cancer cases worldwide in 2016, an increase of about six times compared with a decade ago $(2,3)$. There are some data showing that the younger trend of the incidence of ovarian cancer is becoming increasingly obvious, and patients with ovarian cancer aged under 40 years accounts for about $25.63 \%$ at present (4). It is expected to be the malignant tumor with the highest incidence in gynaecological diseases by 2030 (5). Ovarian cancer not only has an extremely high incidence, but also poses a huge threat to the lives of women. The mortality rate within 5 years of ovarian cancer is $72.8 \%$, being the gynaecological disease with the highest mortality rate (6). In the face of this great threat, ovarian cancer has always been one of the most studied diseases in clinic. With the development of modern medical technology, the 5-year survival rate can be increased to $>70 \%$ if timely treatment can be carried out in the early stage $(7,8)$. However, as the pathogenesis of ovarian cancer is not clear, the current diagnosis of ovarian cancer still needs to be checked by cancer markers and imaging methods, which causes great limitations (9). In recent years, scholars worldwide have begun to focus on the gene with the deepening of research. MicroRNA (miR or miRNA), a conserved non-coding RNA that can horizontally regulate gene expression after transcription has been proven to be closely related to the occurrence and development of human tumor diseases (10-12). miR-26b is a tumor suppressor gene which has been proven to be downregulated in breast cancer and gastric cancer $(13,14)$, but its role in ovarian cancer is not clear. Therefore, in this study, miR-26b was also suspected to have abnormal expression in ovarian cancer and to be closely related to the occurrence and development of ovarian cancer, and experimental analysis was conducted to verify our conjecture, and to provide reference and guidance for clinical diagnosis and treatment of ovarian cancer in the future.

\section{Patients and methods}

General information. A total of 74 patients with ovarian cancer and 30 patients with benign ovarian tumors in the 
Affiliated Hospital of Inner Mongolia Medical University (Hohhot, China) from July 2011 to June 2013 were retrospectively analyzed. All patients were aged 36 to 64 years and with an average age of $48.24 \pm 10.27$ years. Among the patients with ovarian cancer, there were 19 cases with well differentiation, 24 cases with moderate differentiation and 31 cases with poor differentiation; 49 cases with serous ovarian cancer, 25 cases with non-serous ovarian cancer; 27 cases with lymph node metastasis and 47 cases without lymph node metastasis; 12 cases in stage I of pathological stage, 20 cases in stage II, 34 cases in stage III and 8 cases in stage IV. This experiment was approved by the Ethics Committee of the Affiliated Hospital of Inner Mongolia Medical University, and all the above subjects signed the informed consent.

Inclusion and exclusion criteria. Inclusion criteria: i) All patients' clinical symptoms were in accordance with the diagnostic guidelines for ovarian cancer in 2011 (15); ii) Patients diagnosed as ovarian cancer and benign epithelial ovarian tumor by biopsy in the Affiliated Hospital of Inner Mongolia Medical University; iii) Patients aged 30 to 65 years; iv) Patients with complete pathological data; and v) Patients who cooperated with the work arrangement of our medical staff. Exclusion criteria: i) Patients with other tumors; ii) Patients with cardiovascular and cerebrovascular diseases; iii) Patients with severe organ failure; iv) Patients with mental illness; v) Patients with surgical intolerance; vi) Patients receiving no radiotherapy, chemotherapy and hormone therapy before surgery; vii) Patients with physical disabilities; viii) Pregnant patients and ix) Patients undergoing hospital transfer.

Methods. A total of 74 patients with ovarian cancer were treated as the study group and 30 patients with benign ovarian tumor as the control group. Cancer tissues of patients in the two groups were obtained, and the expression of miR-26b (XWCPK3036; Xiamen Yanke Biotechnology Co., Ltd., Fujian, China) was measured by fluorescence reverse transcription-quantitative polymerase chain reaction (RT-qPCR). The miRNA in ovarian cancer tissues was separated and purified strictly according to the instructions of TRIzol RNA isolation kit (15596026; Chengdu Technology \& Market Co., Ltd., Chengdu, China). The miRNA was determined under the sample with OD260/OD280 value of 1.8-2.0, and RNA was reverse transcribed to cDNA according to the specification of the reverse transcription kit (A0005; Shanghai HiFun Biotechnology Co., Ltd., Shanghai, China). At the end of the reaction, the cDNA sample was placed in a refrigerator as a template for the qPCR reaction and the $\mathrm{qPCR}$ primer sequence was synthesized by ThermoFisher Company, (Thermo Fisher Scientific, Inc., Waltham, MA, USA) (Table I). Reaction conditions: cDNA $1 \mu 1$, forward primer $0.4 \mu 1$, universal miRNA qPCR primer $0.4 \mu \mathrm{l}$, 2xTransStart Tip Green qPCR SuperMix $10 \mu \mathrm{l}$, passive reference dye (50x) (Optional) $0.4 \mu \mathrm{l}$, total volume $20 \mu \mathrm{l}$, complement with $\mathrm{ddH}_{2} \mathrm{O}$ to $20 \mu \mathrm{l}$. PCR reaction conditions: 40 cycles of predenaturation at $95^{\circ} \mathrm{C}$ for $5 \mathrm{~min}$, denaturation at $90^{\circ} \mathrm{C}$ for $15 \mathrm{sec}$, annealing and elongation at $60^{\circ} \mathrm{C}$ for $30 \mathrm{sec}$. The relative expression of miR-26b was expressed by $2^{-\Delta \Delta C q}(16)$. All tests were repeated 3 times to obtain the average value.

Observation indicators. i) Clinical data of patients in the two groups (such as age, tumor type and pathological stage); ii) The expression level of miR-26b of patients in the two groups; iii) Relationship between miR-26b and clinicopathology in patients with ovarian cancer; iv) The value of miR-26b in diagnosis of ovarian cancer; and $v$ ) The patients in the experimental group were followed up for 5 years (telephone, mail, hospital re-examination and door-to-door follow-up). The termination date and termination event were June 2018 and the patient's death or loss of contact. According to the median expression of miR-26b in the study group, the patients were divided into two groups: the miR-26b high-expression group and the miR-26b low-expression group. The 5-year survival in the two groups was recorded and compared.

Statistical analysis. SPSS version 22.0 statistical software (Shanghai Yuchuang Network Technology Co., Ltd., Shanghai, China) was used to analyze and process the data. Enumeration data such as the patient's clinical data were expressed in the form of (rate) and Chi-square test was used for comparison among the groups. Measurement data such as the expression of miR-26b was expressed in the form of mean \pm standard deviation. ANOVA was used for multigroup comparison with Dunnett's post hoc test, and t-test was used for comparison between the two groups. ROC curve was used to analyze the diagnostic value. The survival rate was calculated by the Kaplan-Meier method and compared by log-rank test. $\mathrm{P}<0.05$ was considered to indicate a statistically significant difference.

\section{Results}

Comparison of clinical data. There was no significant difference in age, weight, course of disease, marital status, residence, smoking and exercise between the study and control groups $(\mathrm{P}>0.050)$, which indicated that the two groups were comparable (Table II).

Comparison of expression level of miR-26b. The expression level of miR-26b in the study group $(0.28 \pm 0.07)$ was significantly lower than that in the control group $(0.54 \pm 0.11)$ $(\mathrm{P}<0.050$; Fig. 1).

Analysis of correlation between miR-26b and clinicopathology of patients with ovarian cancer. There was no significant correlation between miR-26b expression and age, tumor type, exercise habit, smoking habit of patients with ovarian cancer $(\mathrm{P}>0.050)$, but there was close correlation between miR-26b expression and lymph node metastasis, differentiation degree, pathological stage of patients with ovarian cancer $(\mathrm{P}<0.001$; Table III).

Value of miR-26b in diagnosis of ovarian cancer. ROC curve showed that the AUC was 0.839 , and when the maximum cut-off value was 0.815 , the sensitivity and specificity of miR-26b in diagnosing the ovary was $84.932 \%$ and $77.936 \%$, respectively ( $\mathrm{P}<0.001$; Table IV, Fig. 2).

Prognostic survival. By June 2018, 72 patients in the study group were followed up successfully, with a success rate of $97.30 \%$. According to the median expression of miR-26b in the study group, 0.28 , the patients were divided into two groups: i) miR-26b, the high-expression group (miR-26b expression 
Table I. Primer sequences.

\begin{tabular}{lrr}
\hline Reverse/Forward & miR-26b & U6 \\
\hline Reverse & 5'-CAAAGGTCCATAGCAAGGGT-3' & 5'-CTCGCTTCGGCAGCACA-3' \\
Forward & 5'-GCGACCTTGTCATGGTTTATAG-3' & 5'-AACGCTTCACGAATTTGCGT-3'
\end{tabular}

Table II. Comparison of clinical data [n (\%)].

\begin{tabular}{|c|c|c|c|c|}
\hline Variables & Study group $(n=74)$ & Control group $(n=30)$ & $\chi^{2}$ or t-test & P-value \\
\hline Age & $49.42 \pm 9.68$ & $48.77 \pm 10.24$ & 0.305 & 0.761 \\
\hline Weight (kg) & $59.67 \pm 7.64$ & $60.31 \pm 8.15$ & 0.380 & 0.705 \\
\hline Course of disease (week) & $3.74 \pm 1.04$ & $3.92 \pm 0.86$ & 0.838 & 0.404 \\
\hline Marital status & & & 0.316 & 0.574 \\
\hline Married & $69(93.24)$ & $27(90.00)$ & & \\
\hline Unmarried & $5(6.76)$ & $3(10.00)$ & & \\
\hline Residence & & & 0.152 & 0.697 \\
\hline Urban & $54(72.97)$ & $23(76.67)$ & & \\
\hline Rural & $20(27.03)$ & $7(23.33)$ & & \\
\hline Smoking & & & 0.218 & 0.641 \\
\hline Yes & $48(64.86)$ & $18(60.00)$ & & \\
\hline No & $26(35.14)$ & $12(40.00)$ & & \\
\hline Exercise habit & & & 0.203 & 0.652 \\
\hline Yes & 19 (25.68) & $9(30.00)$ & & \\
\hline No & $55(74.32)$ & $21(70.00)$ & & \\
\hline
\end{tabular}

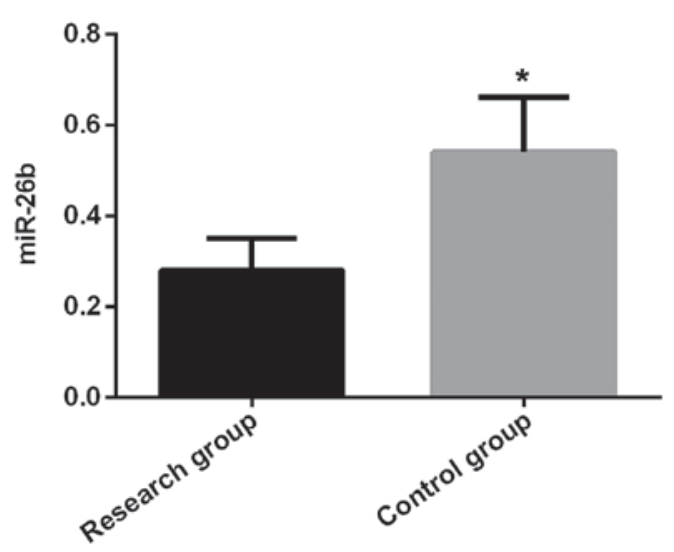

Figure 1. Comparison of expression level of miR-26b. The expression level of miR-26b in the control group was significantly higher than that in the study group $\left({ }^{*} \mathrm{P}<0.050\right)$.

level $<0.28, \mathrm{n}=39$ ); and ii) miR-26b, the low-expression group (miR-26b expression level $\geq 0.28, \mathrm{n}=33$ ). The 5 -year overall survival rate in the low-expression group (61.54\%) was significantly lower than that in the high-expression group (84.85\%) ( $\mathrm{P}=0.028$; Fig. 3).

\section{Discussion}

Ovarian cancer is the malignant tumor with the highest mortality rate in females at present. There is no obvious special symptom in the early stage, which is easy to be ignored or mishandled by the patients. Therefore, most of the patients have reached the middle and late stage at diagnosis, and most of the tumors have metastases and invasion at this time, which greatly increases the difficulty of treatment and makes the prognosis less optimistic $(17,18)$. At present, the pathogenesis of ovarian cancer is not clear, and there is no accurate and effective index for the diagnosis of early ovarian cancer (19). The effective biological index for diagnosis and treatment of ovarian cancer is a hot spot in clinical research at present and with the development of the research, and the discovery of micro-RNA provides a new direction for the modern target research of tumors (20). miRNA is a class of endogenous non-coding small molecules that occupy only 1-3\% of the human genome sequence, with a length of 17-25 nucleotides (21). The incomplete pairing of the non-coding region at the 3'UTR end of its target gene can block the translation of the target gene mRNA and lead to apoptosis, proliferation, metastasis, differentiation and other vital activities with the cells involved in the regulation, which has been continuously proven to play an important role in various tumor diseases as a tumor promoter or tumor suppressor gene $(22,23)$.

miR-26 is composed of four highly conserved small molecules non-coding RNA with similar structures and sequences. miR-26b, a member of miR-26 family, is located on the 4th intron of chromosome 2 dipole CTDSP1, which can inhibit the proliferation and invasion of HCC cells by regulating the expression of EphA2 and c-Myc and has been proven to be 
Table III. Analysis of correlation between miR-26b and clinicopathology of patients with ovarian cancer in the study group.

\begin{tabular}{|c|c|c|c|c|}
\hline Variables & $\mathrm{n}$ & miR-26b level & t or $F$ & P-value \\
\hline Age (years) & & & 0.667 & 0.507 \\
\hline$>49$ & 45 & $0.24 \pm 0.14$ & & \\
\hline$\leq 49$ & 29 & $0.26 \pm 0.10$ & & \\
\hline Lymph node metastasis & & & 10.862 & $<0.001$ \\
\hline Yes & 27 & $0.12 \pm 0.06$ & & \\
\hline No & 47 & $0.35 \pm 0.10$ & & \\
\hline Tumor type & & & 0.527 & 0.600 \\
\hline Serous & 49 & $0.27 \pm 0.07$ & & \\
\hline Non-serous & 25 & $0.26 \pm 0.09$ & & \\
\hline Differentiation degree & & & 35.942 & $<0.001$ \\
\hline Well-differentiated & 19 & $0.38 \pm 0.10$ & & \\
\hline Moderately differentiated & 24 & $0.22 \pm 0.08$ & & \\
\hline Poorly differentiated & 31 & $0.10 \pm 0.14$ & & \\
\hline Pathological stage & & & 14.453 & $<0.001$ \\
\hline Stage I-II & 32 & $0.35 \pm 0.04$ & & \\
\hline Stage III-IV & 42 & $0.15 \pm 0.07$ & & \\
\hline Exercise habit & & & 0.517 & 0.607 \\
\hline Yes & 19 & $0.25 \pm 0.08$ & & \\
\hline No & 55 & $0.24 \pm 0.07$ & & \\
\hline Smoking & & & 0.878 & 0.383 \\
\hline Yes & 48 & $0.26 \pm 0.09$ & & \\
\hline No & 26 & $0.24 \pm 0.10$ & & \\
\hline
\end{tabular}

Table IV. Value of miR-26b in diagnosis of ovarian cancer.

\begin{tabular}{lc}
\hline Indicators & Value \\
\hline Sensitivity & $84.932 \%$ \\
Specificity & $77.936 \%$ \\
Cut-off & 0.815 \\
R & 1.511 \\
$95 \%$ Confidence interval & $1.045-2.185$ \\
Area under curve & 0.839 \\
P & 0.028 \\
\hline
\end{tabular}

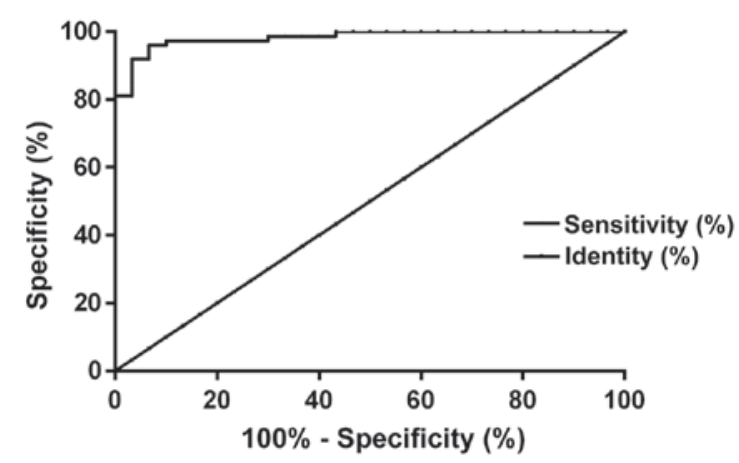

Figure 2. ROC curve analysis of miR-26b in diagnosis of ovarian cancer. ROC curve showed that the AUC was 0.839 , and when the maximum cut-off value was 0.815 , the sensitivity and specificity of miR-26b in diagnosing the ovary was $84.932 \%$ and $77.936 \%$, respectively $(\mathrm{P}<0.001)$.

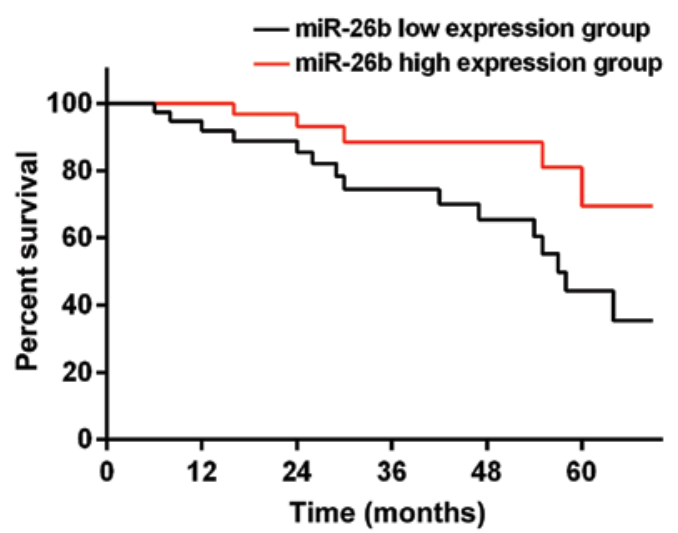

Figure 3. The 5-year survival of patients with ovarian cancer. The 5-year overall survival rate in the low-expression group $(61.54 \%)$ was significantly lower than that in the high-expression group $(84.85 \%), \mathrm{P}=0.028$.

abnormally expressed in many kinds of tumors (23-26). Its role in ovarian cancer has not yet been clarified. Therefore, the expression level of miR-26b in ovarian cancer and benign ovarian epithelial tumor was detected by RT-qPCR technique to explore the abnormal expression of miR-26b in ovarian cancer and its relationship with pathological status of ovarian cancer patients.

The results of this study showed that the expression level of miR-26b in the study group was significantly lower than that in the control group, which indicates that miR-26b is closely related to the occurrence and development of ovarian 
cancer and may be involved in the process of ovarian cancer as proto-oncogene. This is also consistent with the results of Liu et al (27), thus supporting this experiment. Further analysis of the differences of miR-26b in clinicopathology of ovarian cancer patients revealed that there was no significant correlation between miR-26b expression and age, tumor type, exercise habit, smoking habit of patients with ovarian cancer ( $P>0.050)$, but there was a close correlation between miR-26b expression and pathological stage, differentiation degree, lymph node metastasis of patients with ovarian cancer, which suggests that miR-26b is closely related to the differentiation and proliferation of ovarian cancer cells and the degree of disease deterioration can be judged by detecting the expression level of miR-26b in patients. ROC curve showed that, when the maximum cut-off value was 0.815 , the sensitivity and specificity of miR-26b in diagnosing the ovary was $84.932 \%$ and $77.936 \%$, respectively, which shows that miR-26b has high diagnostic value and is expected to be an effective target for the future diagnosis of ovarian cancer. Comparison of prognostic 5 -year survival between the miR-26b high-expression and miR-26b low-expression groups showed that the 5-year overall survival rate in the low-expression group was significantly lower than that in the high-expression group, which indicates that miR-26b is closely related to the prognosis of ovarian cancer patients and can be used as an effective biomarker for monitoring the prognosis of ovarian cancer patients.

At present, the diagnosis of ovarian cancer still needs to be confirmed by tissue biopsy that can cause great damage to the body and is still difficult for early diagnosis of ovarian cancer. Therefore, a convenient and effective biomarker is urgently needed to diagnose and treat ovarian cancer and miRNA is a substance that can be detected in serum, plasma, body fluids and tissues, so it has become a major focus of oncologists worldwide. In this study, miR-26b was confirmed to be an effective marker for the diagnosis and treatment of ovarian cancer. However, due to the limited experimental conditions, there are still some shortcomings. For example, the number of subjects is relatively small, and the mechanism of miR-26b in ovarian cancer is not clear, which needs further study.

In conclusion, miR-26b is under-expressed in ovary and has a close relationship with the pathological stage, differentiation degree, and lymph node metastasis of ovarian cancer, which indicates that miR-26b is involved in the occurrence and development of ovarian cancer and is expected to be an effective indicator for treatment and diagnosis of ovarian cancer and the prognosis of patients.

\section{Acknowledgements}

Not applicable.

\section{Funding}

No funding was received.

\section{Availability of data and materials}

The datasets used and/or analyzed during the present study are available from the corresponding author on reasonable request.

\section{Authors' contributions}

JL, WZ and YD designed the study and helped with observation indicators. JL, XL and JS collected and interpreted the patients' general data and were responsible for statistical analysis. All authors read and approved the final manuscript.

\section{Ethics approval and consent to participate}

The study was approved by the Ethics Committee of the Affiliated Hospital of Inner Mongolia Medical University (Hohhot, China). Patients who participated in this study, had complete clinical data. Signed informed consents were obtained from the patients or guardians.

\section{Patient consent for publication}

Not applicable.

\section{Competing interests}

The authors declare that they have no competing interests.

\section{References}

1. Jaaback K and Johnson N: Intraperitoneal chemotherapy for the initial management of primary epithelial ovarian cancer. Cochrane Database Syst Rev 1: CD005340, 2006.

2. Mirza MR, Monk BJ, Herrstedt J, Oza AM, Mahner S, Redondo A, Fabbro M, Ledermann JA, Lorusso D, Vergote I, et al: ENGOT-OV16/NOVA investigators: Niraparib maintenance therapy in platinum-sensitive, recurrent ovarian cancer. $\mathrm{N}$ Engl J Med 375: 2154-2164, 2016.

3. Jacobs IJ, Menon U, Ryan A, Gentry-Maharaj A, Burnell M, Kalsi JK, Amso NN, Apostolidou S, Benjamin E, Cruickshank D, et al: Ovarian cancer screening and mortality in the UK collaborative trial of ovarian cancer screening (UKCTOCS): A randomised controlled trial. Lancet 387: 945-956, 2016.

4. Oikonomopoulou K, Li L, Zheng Y, Simon I, Wolfert RL, Valik D, Nekulova M, Simickova M, Frgala T and Diamandis EP: Prediction of ovarian cancer prognosis and response to chemotherapy by a serum-based multiparametric biomarker panel. Br J Cancer 99 :1103-1113, 2008.

5. Zhang H, Liu T, Zhang Z, Payne SH, Zhang B, McDermott JE, Zhou JY, Petyuk VA, Chen L, Ray D, et al: CPTAC investigators: Integrated proteogenomic characterization of human high-grade serous ovarian cancer. Cell 166: 755-765, 2016.

6. Wright AA, Bohlke K, Armstrong DK, Bookman MA, Cliby WA, Coleman RL, Dizon DS, Kash JJ, Meyer LA, Moore KN, et al: Neoadjuvant chemotherapy for newly diagnosed, advanced ovarian cancer: Society of gynecologic oncology and American society of clinical oncology clinical practice guideline. Gynecol Oncol 143: 3-15, 2016.

7. Zhao Z, Yang Y, Zeng Y and He M: A microfluidic ExoSearch chip for multiplexed exosome detection towards blood-based ovarian cancer diagnosis. Lab Chip 16: 489-496, 2016.

8. Strickland KC, Howitt BE, Shukla SA, Rodig S, Ritterhouse LL, Liu JF, Garber JE, Chowdhury D, Wu CJ, D'Andrea AD, et al: Association and prognostic significance of BRCA1/2-mutation status with neoantigen load, number of tumor-infiltrating lymphocytes and expression of PD-1/PD-L1 in high grade serous ovarian cancer. Oncotarget 7: 13587-13598, 2016.

9. Au Yeung CL, Co NN, Tsuruga T, Yeung TL, Kwan SY, Leung CS, Li Y, Lu ES, Kwan K, Wong KK, et al: Exosomal transfer of stroma-derived miR21 confers paclitaxel resistance in ovarian cancer cells through targeting APAF1. Nat Commun 7: 11150, 2016.

10. Bracken CP, Scott HS and Goodall GJ: A network-biology perspective of microRNA function and dysfunction in cancer. Nat Rev Genet 17: 719-732, 2016. 
11. Rupaimoole R and Slack FJ: MicroRNA therapeutics: Towards a new era for the management of cancer and other diseases. Nat Rev Drug Discov 16: 203-222, 2017.

12. Christopher AF, Kaur RP, Kaur G, Kaur A, Gupta V and Bansal P: MicroRNA therapeutics: Discovering novel targets and developing specific therapy. Perspect Clin Res 7: 68-74, 2016.

13. Li D, Wei Y, Wang D, Gao H and Liu K: MicroRNA-26b suppresses the metastasis of non-small cell lung cancer by targeting MIEN1 via NF- $\kappa \mathrm{B} / \mathrm{MMP}-9 / \mathrm{VEGF}$ pathways. Biochem Biophys Res Commun 472: 465-470, 2016.

14. John Clotaire DZ, Zhang B, Wei N, Gao R, Zhao F, Wang Y, Lei M and Huang W: MiR-26b inhibits autophagy by targeting ULK2 in prostate cancer cells. Biochem Biophys Res Commun 472: 194-200, 2016.

15. Burges A and Schmalfeldt B: Ovarian cancer: Diagnosis and treatment. Dtsch Arztebl Int 108: 635-641, 2011.

16. Livak KJ and Schmittgen TD: Analysis of relative gene expression data using real time quantitative PCR and the 2(-Delta Delta C(T)) method. Methods 25: 402-408, 2001.

17. Wentzensen N, Poole EM, Trabert B, White E, Arslan AA, Patel AV, Setiawan VW, Visvanathan K, Weiderpass E, Adami HO, et al: Ovarian cancer risk factors by histologic subtype: An analysis from the ovarian cancer cohort consortium. J Clin Oncol 34: 2888-2898, 2016.

18. Romagnolo C, Leon AE, Fabricio ASC, Taborelli M, Polesel J, Del Pup L, Steffan A, Cervo S, Ravaggi A, Zanotti L, et al: HE4, CA125 and risk of ovarian malignancy algorithm (ROMA) as diagnostic tools for ovarian cancer in patients with a pelvic mass: An Italian multicenter study. Gynecol Oncol 141: 303-311, 2016.

19. Morgan RJ Jr, Armstrong DK, Alvarez RD, Bakkum-Gamez JN, Behbakht K, Chen LM, Copeland L, Crispens MA, DeRosa M, Dorigo O, et al: Ovarian Cancer, Version 1.2016, NCCN Clinical Practice Guidelines in Oncology. J Natl Compr Canc Netw 14: 1134-1163, 2016.

20. Inamura $\mathrm{K}$ and Ishikawa Y: MicroRNA in lung cancer: Novel biomarkers and potential tools for treatment. J Clin Med 5: 36 , 2016.
21. Saadatpour L, Fadaee E, Fadaei S, Nassiri Mansour R, Mohammadi M, Mousavi SM, Goodarzi M, Verdi J and Mirzaei H: Glioblastoma: Exosome and microRNA as novel diagnosis biomarkers. Cancer Gene Ther 23: 415-418, 2016.

22. Li Z, Ma YY, Wang J, Zeng XF, Li R, Kang W and Hao XK: Exosomal microRNA-141 is upregulated in the serum of prostate cancer patients. Onco Targets Ther 9: 139-148, 2015.

23. Sun C, Li S, Yang C, Xi Y, Wang L, Zhang F and Li D: MicroRNA-187-3p mitigates non-small cell lung cancer (NSCLC) development through down-regulation of BCL6. Biochem Biophys Res Commun 471: 82-88, 2016.

24. Rubiś P, Totoń-Zurańska J, Wiśniowska-Śmiałek S, Holcman K, Kołton-Wróż M, Wołkow P, Wypasek E, Natorska J, Rudnicka-Sosin L, Pawlak A, et al: Relations between circulating microRNAs (miR-21, miR-26, miR-29, miR-30 and miR-133a), extracellular matrix fibrosis and serum markers of fibrosis in dilated cardiomyopathy. Int J Cardiol 231: 201-206, 2017.

25. Li Y, Sun Z, Liu B, Shan Y, Zhao L and Jia L: Tumor-suppressive miR-26a and miR-26b inhibit cell aggressiveness by regulating FUT4 in colorectal cancer. Cell Death Dis 8: e2892, 2017.

26. Shi L, Yin W, Zhang Z and Shi G: Down-regulation of miR-26b induces cisplatin resistance in nasopharyngeal carcinoma by repressing JAG1. FEBS Open Bio 6: 1211-1219, 2016.

27. Liu J, Tu F, Yao W, Li X, Xie Z, Liu H, Li Q and Pan Z: Conserved miR-26b enhances ovarian granulosa cell apoptosis through HAS2-HA-CD44-Caspase-3 pathway by targeting HAS2. Sci Rep 6: 21197, 2016.

(i) $($ This work is licensed under a Creative Commons Attribution-NonCommercial-NoDerivatives 4.0 International (CC BY-NC-ND 4.0) License. 